\title{
Re-expression of tumorigenicity after attenuation of human synaptotagmin 13 in a suppressed microcell hybrid cell line
}

\author{
JENNIFER E. JAHN ${ }^{1}$ and WILLIAM B. COLEMAN ${ }^{2}$ \\ ${ }^{1}$ Department of Cell and Molecular Pharmacology, Medical University of South Carolina, 173 Ashley Avenue, \\ Charleston, SC 29245; ${ }^{2}$ Department of Pathology and Laboratory Medicine, Curriculum in Toxicology, \\ Program in Translational Medicine, UNC Lineberger Comprehensive Cancer Center, \\ University of North Carolina School of Medicine, Chapel Hill, NC 27713, USA
}

Received August 31, 2007; Accepted October 22, 2007

\begin{abstract}
Human chromosome 11p11.2 contains a putative liver tumor suppressor locus that was identified using a microcell hybrid cell line-based model of tumor suppression. Transcription mapping of suppressed microcell hybrid cell lines suggests that human SYT13 represents a strong candidate for the $11 \mathrm{p} 11.2$ tumor suppressor gene. Other evidence suggests that the putative $11 \mathrm{p} 11.2$ tumor suppressor gene (SYT13 or some other) may modulate the tumorigenic potential of neoplastic liver cell lines through direct or indirect regulation of the rat Wtl tumor suppressor gene. To characterize a functional role for SYT13 in liver tumor suppression, we employed RNAi to attenuate SYT13 expression in a suppressed microcell hybrid cell line (GN6TF-1 1 $\left.{ }^{\text {neo }} \mathrm{CX} 4\right)$. SYT13-attenuated cells display aggressive phenotypic properties that are similar to or indistinguishable from the parental tumor cells (GN6TF), including altered cellular morphologies, disrupted contact inhibition, elevated saturation densities, restoration of anchorage-independent growth and increased tumorigenicity in vivo. Moreover, SYT13 attenuation and re-expression of tumorigenicity in GN6TF- $11^{\text {neo }} \mathrm{CX} 4$-derived cell lines was accompanied by a significant decrease of $W t l$ expression. In contrast, the phenotypic properties of scrambled-control cells were similar to the suppressed microcell hybrid cells and
\end{abstract}

Correspondence to: Dr William B. Coleman, Department of Pathology and Laboratory Medicine, University of North Carolina School of Medicine, 515 Brinkhous-Bullitt Building, CB\# 7525, Chapel Hill, NC 27599, USA

E-mail: william.coleman@pathology.unc.edu

Abbreviations: SYT13, synaptotagmin 13; hSYT13, human SYT13; rSyt13, rat Syt13; PRDM11, PR domain containing 11; TP53I11, tumor protein p53 inducible protein 11 ; Wt1, Wilms' tumor 1; RNAi, RNA interference; shRNA, short-hairpin RNA; RT-PCR, reverse-transcription polymerase chain reaction

Key words: hepatocellular carcinoma, human 11p11.2, microcell hybrid, RNAi, SYT13, tumor suppressor gene
Wt 1 expression was unaffected. These observations combine to establish that: i) human SYT13 functions as a liver tumor suppressor gene that complements a molecular defect in GN6TF rat liver tumor cells resulting in a normalized cellular phenotype in vitro and suppression of tumorigenicity in vivo; ii) RNAi-mediated attenuation of SYT13 expression restores the neoplastic phenotype of GN6TF- $11^{\text {neo }} \mathrm{CX} 4$ microcell hybrid cells, consistent with the function of a liver tumor suppressor gene; and iii) loss of $W t 1$ expression is important for the re-establishment of tumorigenic potential by GN6TF$11^{\text {neo }} \mathrm{CX} 4$ microcell hybrid cells after attenuation of SYT13.

\section{Introduction}

Hepatocellular carcinogenesis is a multi-step process that results from altered expression of multiple genes as a consequence of genetic mutations, epigenetic mechanisms, and/or chromosomal aberrations affecting a number of chromosome arms $(1,2)$, including chromosome 11p (3-6). Comparative mapping studies suggest strongly that hepatocarcinogenesis in humans and rodents may be governed by orthologous tumor suppressor genes (7). We exploited this supposition to identify human liver tumor suppressor genes using a functional model for tumor suppression based on microcell-mediated transfer of human chromosome 11 into the aggressive rat liver tumor cell line GN6TF (8). The resulting microcell hybrid cell lines exhibit normalized cellular morphology, re-expression of contact inhibition, complete loss of anchorage-independent growth potential, and decreased tumorigenicity in vivo, suggesting that human chromosome 11 contains one or more liver tumor suppressor genes (8). Genomic mapping localized the liver tumor suppressor locus to a small $(<1 \mathrm{Mbp})$ region of human chromosome $11 \mathrm{p} 11.2$ containing a number of candidate genes $(9,10)$. Subsequently, transcription mapping identified several candidate $11 \mathrm{p} 11.2$ tumor suppressor genes (including TP53I11, PRDM11, LOC219638 and SYT13) that were expressed in a panel of suppressed microcell hybrid cell lines $(11,12)$. SYT13 mRNA was detected in $100 \%$ of suppressed microcell hybrid cell lines and was absent in all non-suppressed clones, including a non-suppressed microcell hybrid cell line that contains chromosome 11 with an 
interstitial deletion at the suppressive $11 \mathrm{p} 11.2$ locus $(11,13)$. Finally, SYT13 expression was lost in all microcell hybrid-derived tumor cell lines examined, coordinate with reexpression of the tumorigenic phenotype (11). Based on its differential expression among microcell hybrid cell lines, SYT13 emerged as an excellent candidate for the human 11 p11.2 liver tumor suppressor gene. The mechanism of SYT13-mediated tumor suppression in this model system is not yet known. However, induction of rat Wt1 gene expression accompanies suppression of tumorigenicity by human 11p11.2 (14), suggesting that the 11p11.2 liver tumor suppressor gene (SYT13 or some other gene) may directly or indirectly regulate $W t 1$ gene expression. Based upon the results of these studies, we propose that: i) SYT13 represents an excellent candidate for the human 11p11.2 liver tumor suppressor gene; and ii) the tumor suppressor function of SYT13 may be mediated through (or require) expression of the rat Wtl gene.

To characterize the mechanistic role of SYT13 in this model of liver tumor suppression, we analyzed phenotypic alterations in the suppressed microcell hybrid cell line GN6TF- $11^{\text {neo }} \mathrm{CX} 4$ after stable silencing of human SYT13 utilizing RNA interference (RNAi). Cells were transfected with an expression vector encoding a SYT13-specific short hairpin dsRNA (shRNA), resulting in a severe attenuation of SYT13 protein expression. SYT13-targeted cell lines display aggressive phenotypic properties similar to the parental GN6TF tumor cells, consistent with the suggestion that SYT13 functions as a tumor suppressor in this model system. Cell morphologies were altered, saturation densities were elevated, anchorage-independent growth was restored, and tumorigenicity in vivo was significantly increased. In addition, rat $W t 1$ gene expression levels were depressed in SYT13-attenuated cell lines, suggesting that loss of Wt1 may contribute to the expression of the aggressive phenotypic characteristics. In contrast, cell lines expressing a scrambled control SYT13 shRNA retain a robust level of Wt1 expression and display a phenotype both in vitro and in vivo similar to that of the suppressed GN6TF- $11^{\text {neo }} \mathrm{CX} 4$ cells. These observations combine to establish that: i) human SYT13 functions as a liver tumor suppressor gene that complements a molecular defect in GN6TF rat liver tumor cells resulting in a normalized cellular phenotype in vitro and suppression of tumorigenicity in vivo; ii) RNAi-mediated attenuation of SYT13 expression restores the neoplastic phenotype of GN6TF-11 ${ }^{\text {neo }} \mathrm{CX} 4$ microcell hybrid cells, consistent with the function of a liver tumor suppressor gene; and iii) loss of Wt1 expression is important for the re-establishment of tumorigenic potential by $\mathrm{GN} 6 \mathrm{TF}-11^{\text {neo }} \mathrm{CX} 4$ microcell hybrid cells after attenuation of $S Y T 13$, consistent with the possibility that WtI is the ultimate tumor suppressor gene in this model system.

\section{Materials and methods}

Cell lines and culture. The rat liver epithelial tumor cell line GN6TF (15) was derived from a normal rat liver epithelial cell line (termed WB-F344) that has been described previously (16). Microcell hybrid cell lines were established from GN6TF rat liver tumor cells $(8,13)$ by microcell- mediated chromosome transfer of human chromosome 11, using established methods $(17,18)$. Clonal GN6TF-11 neo microcell hybrid cell lines used for chromosome deletion and transcription mapping have been described (11). In the current study, GN6TF- $11^{\text {neo }} \mathrm{CX} 4$ cells were transfected with shRNA expression constructs (as described below). All phenotypic and gene expression-related comparisons were made relative to GN6TF tumor cells and GN6TF-11 ${ }^{\text {neo }} \mathrm{CX} 4$ microcell hybrid cells. All cell lines were cultured in Richter's minimal essential medium supplemented as described (19). GN6TF- $11^{\text {neo }} \mathrm{CX} 4$ cells (and derived cell lines) were maintained in medium containing $800 \mu \mathrm{g} / \mathrm{ml}$ G418 (8). After transfection with shRNA vectors, cell lines derived from GN6TF- $11^{\text {neo }} \mathrm{CX} 4$ cells were maintained in medium containing $180 \mu \mathrm{g} / \mathrm{ml}$ Zeocin (InvivoGen, San Diego, CA).

Short hairpin-RNA expression vectors. Expression vectors for SYT13 shRNA (and scrambled control shRNAs) were constructed using psiRNA-hH1zeo (InvivoGen). This plasmid contains a BbsI cloning site downstream of the H1 promoter which drives the expression of a short dsRNA hairpin structure, as well as an Sh Ble expression cassette (under the control of the EM7 promoter) which confers resistance to Zeocin. Targeting oligodeoxynucleotides (designated SYT13-i), designed according to guidelines provided by InvivoGen, were directed against a 21 base pair target in exon 2 of hSYT13 (Fig. 1) which has 71\% (15/21) sequence identity to the rat $S y t 13$ gene. Control oligodeoxynucleotides (designated SYT13-s) were generated by scrambling nucleotides of the SYT13-i targeting sequence (SYT13-i, 5'-AGA CTA TTC ACT GAG GTC TAC-3'; SYT13-s, 5'-A

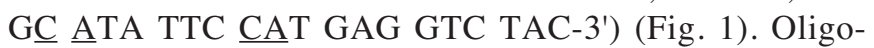
deoxynucleotides corresponding to sense and anti-sense strands for both the SYT13-i and SYT13-s sequences were synthesized by the UNC Oligodeoxynucleotide Core Facility (Chapel Hill, NC). These oligodeoxynucleotides were denatured, annealed, cloned into the psiRNA-hH1zeo plasmid and propagated in E. coli GT116 (InvivoGen). Plasmids were purified using the Wizard Midiprep kit from Qiagen (Madison, WI) and sequenced by the UNC DNA Core Sequencing Facility to verify the construction of the shRNA expression cassettes.

Production of stably transfected SYT13-targeted and control cell clones. Transfection and stable integration of DNA vectors into the $\mathrm{GN} 6 \mathrm{TF}-11^{\text {neo }} \mathrm{CX} 4$ cell line was achieved using Lipofectamine 2000 (Invitrogen, Carlsbad, CA) according to the protocol provided by the manufacturer. Purified plasmid DNA $(5 \mu \mathrm{g})$ was linearized by SwaI digestion and then transfected into $8.6 \times 10^{5}$ cells. Successfully transfected cells were selected in $180 \mu \mathrm{g} / \mathrm{ml}$ Zeocin (InvivoGen), and individual cell lines (designated with the prefix SYT13-i and SYT13-s, respectively) were clonally isolated and maintained in culture as previously described (11).

Gene expression analysis. Transcriptional analyses of SYT13-i and SYT13-s cell lines included semi-quantitative RT-PCR and/or quantitative real-time RT-PCR for various gene targets. Total cellular RNA was isolated from cultured 


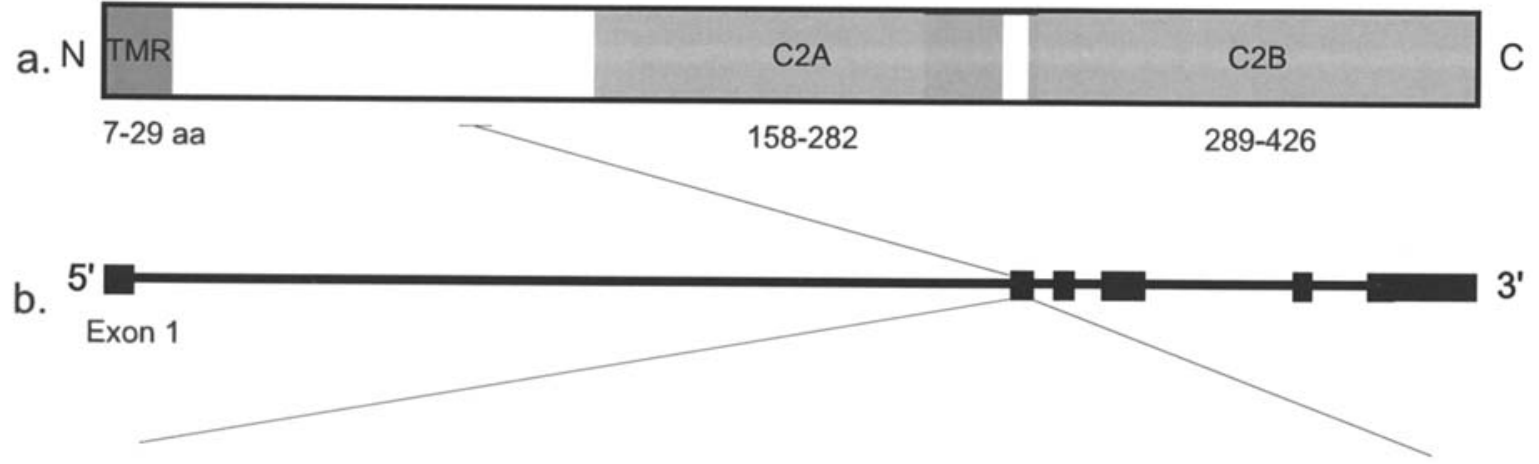
TTCAATGTTAAAAAGTCCACGGAACCTGTTCAGCCCCGTGCCCTCCTCAAGTTCCCAGACATCTATGGACCC
AGGCCAGCTGTGACGGCTCCAGAGGTCATCAACTATGCAGACTATTCACTGAGGTCTACGGAGGAGCCCACT
GCACCTGCCAGCCCCCAACCCCGAATGACAGTCGCCTCAAGAGGCAGGTCACAGAGGAGCTGTTCATCCTC
CCTCAGAATG

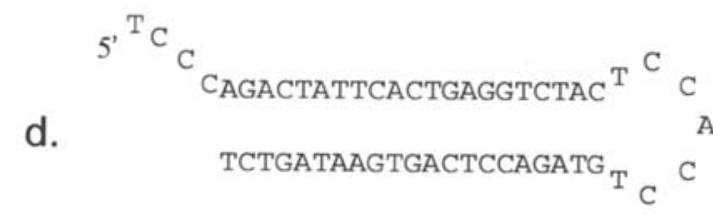

SYT13i shRNA

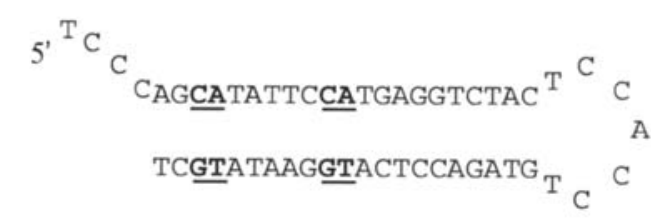

SYT13s shRNA

Figure 1. Design of SYT13-i and SYT13-s short hairpin RNAs. (a) The SYT13 protein contains an N-terminal transmembrane region (designated TMR) followed by a cytoplasmic linker region and two tandem C2 domains (designated C2A and C2B). (b) The SYT13 gene is comprised of 6 exons. The correspondence between the exon structure and the protein structure of SYT13 is indicated. (c) The 21 nucleotide SYT13 target localizes to the coding sequence of exon 2, which corresponds to the linker region of the protein. The target sequence is indicated in bold/underlined. (d) The sequence and structure of SYT13-i and SYT13-s shRNAs. The four scrambled nucleotides of SYT13-s are shown in bold/underlined.

cells using RNeasy (Qiagen, Valencia, CA) according to the manufacturer's guidelines. For quantitative reverse transcription-PCR, oligodeoxynucleotides to rat Wt1 were designed around intron-exon boundaries using Primer Express software (Perkin-Elmer Applied Biosystems, Foster City, CA). Each multiplex real-time RT-PCR was carried out in triplicate in a $30 \mu 1$ volume [2X ABsolute QPCR ROX Mix (ABgene, Rochester, NY), $0.1 \mu \mathrm{g} / \mu 1$ of each primer, $20 \mu \mathrm{M}$ TaqMan probe, 5 U Reverse-iT Rtase Blend (ABgene), $100 \mathrm{ng}$ total RNA] for $30 \mathrm{~min}$ at $48^{\circ} \mathrm{C}$ for reverse transcription, $10 \mathrm{~min}$ at $95^{\circ} \mathrm{C}$ for initial denaturing, followed by 40 cycles of $95^{\circ} \mathrm{C}$ for $15 \mathrm{sec}$ and $60^{\circ} \mathrm{C}$ for $1 \mathrm{~min}$ in the ABI PRISM 7700 Sequence Detection System (PerkinElmer Applied Biosystems). Values for Wt1 expression were subtracted from the same-tube expression of Gapdh and relative quantitation of the individual reactions were normalized to GN6TF- $11^{\text {neo }} \mathrm{CX} 4$ mRNA using the Comparative $\left(2^{\Delta \Delta \mathrm{Ct}}\right)$ method described in User Bulletin \#2: ABI Prism 7700 Sequence Detection System. The following primers and probes were used: rat Gapdh forward, 5'-ATG GGT GTG AAC CAC GAG AA-3', reverse, 5'-GGC ATG GAC TGT GGT CAT GA-3', probe, 5'-tet TGC ATC CTG CAC CAC CAA CTG CTT AG-3' tamra, and rat Wt1 forward, 5'-TCT TCC GAG GCA TTC AGG AT-3', reverse, 5'-TTC AGA TGC TGA CCG GAC AA-3', probe, 5'-fam CCA CTC CAG ATA CGC GCC GCA-3' tamra. Semiquantitative RT-PCR was performed as described previously (11). RNA (1 $\mu \mathrm{g})$ was reversed-transcribed with RETRO script (Ambion, Austin, TX). PCR reactions consisted of
$2 \mu 1 \mathrm{RT}$ reaction in $50 \mu 1$ of a buffer consisting of $50 \mathrm{mM}$ $\mathrm{KCl}, 20 \mathrm{mM}$ Tris- $\mathrm{HCl}$ (pH 8.4), $2 \mathrm{mM} \mathrm{MgCl}_{2}, 200 \mu \mathrm{M}$ of each dNTP (EasyStart PCR Mix-in-a-Tube, Molecular BioProducts, San Diego, CA), 0.4 $\mu \mathrm{M}$ of each primer, and $2.5 \mathrm{U}$ AmpliTaq enzyme (Perkin-Elmer Applied Biosystems). Amplifications were carried out in a Perkin-Elmer Thermocycler using a step-cycle program consisting of 30 cycles of $94^{\circ} \mathrm{C}$ for denaturing $(1 \mathrm{~min}), 55-65^{\circ} \mathrm{C}$ for annealing $(1 \mathrm{~min})$ and $72^{\circ} \mathrm{C}$ for extension $(2 \mathrm{~min})$. PCR products were fractionated on $2 \%$ agarose gels containing $40 \mathrm{mM}$ Trisacetate/1 mM EDTA ( $\mathrm{pH} 8.0$ ) and visualized by ethidium bromide staining. The following primers were used: hSYT13 forward, 5'-TTA ACA ATG TGG ACA TCT GTT TAGA-3', reverse, 5'-TTA GTC TAT GAC ATC TGG CTA CATG-3' (11); PRDM11 forward, 5'-CTT GGG GAT GAC CTC GTT TA-3', reverse, 5'-AAA GCT TCC AGC AAG TGG AC-3'; TP53I11 forward, 5'-TGT GGA ACG CTC TCT ACA CG-3', reverse 5'-TTC GGC CGA CTT GGT AAT AG-3'; and B-actin forward, 5'-AGA GAT GGC CAC GGC TGC TT-3', reverse, 5'-ATT TGC GGT GGA CGA TGG AG-3' (11). Unless otherwise noted, oligodeoxynucleotide primers were designed using the Primer3 PCR primer program (http://frodo.wi.mit.edu/) (20).

Affymetrix microarray analysis. Large-scale gene expression analysis was performed by Expression Analysis (Durham, NC) (www.expressionanalysis.com) using the Affymetrix GeneChip Rat Genome 230 2.0 Array (Affymetrix, Santa Clara, CA). Target RNA was prepared and hybridized 
according to the Affymetrix Technical Manual as previously described (21) and statistical analysis was performed by Expression Analysis proprietary methods. Expression levels of select genes (including interferon-associated genes and potential off-target genes) were mined from the microarray data.

Western blot analysis. A rabbit polyclonal antibody directed against hSYT13 was generated by Anaspec (San Jose, CA). The antibody was raised against a 14 amino acid sequence (RDQDPDLEKAKPSL) located in the linker region between the transmembrane and $\mathrm{C} 2 \mathrm{~A}$ domains of hSYT13 (corresponding to amino acids 41-57). This peptide target was chosen based on its antigenicity and divergence from the rSyt13 homolog $(57 \%, 8 / 14$ amino acids). Rabbits $(n=2)$ were initially immunized with $4 \mathrm{mg}$ KLH-conjugated peptide in Complete Freund's Adjuvant, and subsequently immunized at 3, 6 and 10 weeks with the KLH-conjugated peptide in incomplete Freund's Adjuvant. Serum was collected 7 and 11 weeks after the initial immunization.

Cells intended for Western blot analysis were lysed in RIPA buffer [50 mM Tris- $\mathrm{HCl}$ (pH 8.0), $150 \mathrm{mM} \mathrm{NaCl}$, $1 \%$ NP-40, $0.5 \%$ sodium deoxycholate, $0.1 \%$ SDS] and subjected to Western analysis using standard methods. Protein concentrations were determined using the BCA protein assay (Pierce, Rockford, IL). Lysates (50 $\mu \mathrm{g}$ protein) were fractioned by SDS-PAGE on $10 \%$ polyacrylamide resolving gels and transferred to nitrocellulose membrane (BA85, $0.45 \mu \mathrm{m}$ pore size, Schleicher \& Schuell, Keene, $\mathrm{NH})$. The hSYT13 antibody was used at a dilution of 1:1000 in 5\% milk/TBST [10 mM Tris-HCl (pH 8.0), $0.15 \mathrm{M} \mathrm{NaCl}$, $0.05 \%$ Tween-20]. Bound primary antibody was detected using a goat anti-rabbit IgG peroxidase conjugate (Sigma Chemical Company, St. Louis, MO, A-9169; 1:10,000) and visualized by chemiluminescence using the Amersham ECL kit (GE Healthcare, Buckinghamshire, UK).

Phenotypic characterization of shRNA-transfected cell lines. The morphologies of cells in culture were evaluated by phase contrast microscopy. For determination of saturation densities in monolayer cultures, cells were plated at a density of $1.25 \times 10^{5}$ cells $/ 60-\mathrm{mm}$ dish and maintained in culture with medium changes every 3 days. At the end of 14 days, cells were harvested and counted using a Model Z1 particle counter (Beckman Coulter, Fullerton, CA). Anchorage-independent growth was assayed as described previously (15). To determine tumorigenicity of the cell lines, suspensions containing $1 \times 10^{6}$ cells were injected into dorsal subcutaneous tissue of 1-day-old Fischer 344 rats (Charles River Laboratories, Inc., Wilmington, MA). Animals were euthanized when tumors reached an approximate diameter of $1 \mathrm{~cm}$ or after 10 months. Studies involving the use of animals were carried out in accordance with federal and institutional guidelines put forth by the National Institutes of Health and the Institutional Animal Care and Use Committee of the University of North Carolina at Chapel Hill.

Statistical analysis. Data in the text, table and figures represent mean values \pm the standard error of the mean.
Statistical analysis of real-time PCR data was accomplished using one-way ANOVA (KaleidaGraph, Reading, PA). The statistical significance of linear correlation coefficients was calculated using VasserStats Significance of Correlation Coefficient Calculator (http://faculty.vasser.edu/lowry/ rsig.html). The statistical significance of data from saturation density and anchorage-independent growth assays was assessed using the Student's t-test for unpaired data with unequal variance (KaleidaGraph). Statistical significance associated with Kaplan-Meier survival analysis was determined using the log-rank test (GraphPad Prism, San Diego, CA).

\section{Results}

SYT13 expression in SYT13-i and SYT13-s cell lines. Introduction of human chromosome 11 into the tumorigenic GN6TF cell line resulted in suppressed microcell hybrid cell lines, including GN6TF-1 $1{ }^{\text {neo }} C X 4$ (8). Selected GN6TF$11^{\text {neo }} \mathrm{CX} 4$-derived transfected cell lines that carry the psiRNA vectors for SYT13-targeting (designated SYT13-i3, SYT13-i5, SYT13-i6 and SYT13-i12) or scrambled-control (designated SYT13-s1 and SYT13-s8) were expanded and SYT13 knockdown was assessed by Western blot. The SYT13 protein is approximately $66 \mathrm{kDa}$ in size $(22,23)$, but synaptotagmin protein family members are known to form SDS-resistant dimers (24). Both monomeric and dimeric forms of immunoreactive hSYT13 protein were detected in GN6TF-1 $1{ }^{\text {neo }} \mathrm{CX} 4$ SYT13-i and SYT13-s cell lines using anti-SYT13 antibodies (Fig. 2a). The anti-SYT13 antibody was designed for human SYT13 specificity, but recognizes the rat Syt13 protein in GN6TF cells (Fig. 2a). GN6TF cells express significantly reduced levels of rSyt13 protein compared to GN6TF$11^{\text {neo }} \mathrm{CX} 4$ cells, and there is a complete absence of the dimeric form of the protein. The amount of SYT13 detected in GN6TF-1 $11^{\text {neo }} \mathrm{CX} 4$ cells reflects the contribution of the human allele in the microcell hybrid cell line. All SYT13-i cell lines examined (SYT13-i3, SYT13-i5, SYT13-i6 and SYT13-i12) express less $S Y T 13$ protein than the GN6TF-11 $1{ }^{\text {neo }} \mathrm{CX} 4$ cells (Fig. 2a). The diminished SYT13 protein level among these cell lines was most pronounced when the dimeric form of the protein is considered (Fig. 2a). In contrast, the SYT13-s cell lines examined (SYT13-s1 and SYT13-s8) express levels of SYT13 protein that are similar or indistinguishable from that observed in GN6TF-1 ${ }^{\text {neo }} \mathrm{CX} 4$ cells (Fig. 2a).

Short hairpin RNA-mediated interferon response and potential off-target effects. Transfection of cells with shRNA can result in an interferon response (25-27) or offtarget effects (28). In studies that have shown an interferon response to shRNA transfection, numerous interferonassociated genes were found to be induced 2- to 5-fold (27). The gene expression profile of both SYT13-i and SYT13-s cell lines (including SYT13-i3, SYT13-i5, SYT13-i12 and $S Y T 13$-s1) were evaluated by microarray analysis to determine the levels of expression of 35 interferon-associated genes. There was minimal change in gene expression for these genes among SYT13-i and SYT13-s cells when compared to GN6TF- $11^{\text {neo }} \mathrm{CX} 4$ cells; 34/35 (97\%) interferon-associated genes were expressed at levels equal to or less than that 
a

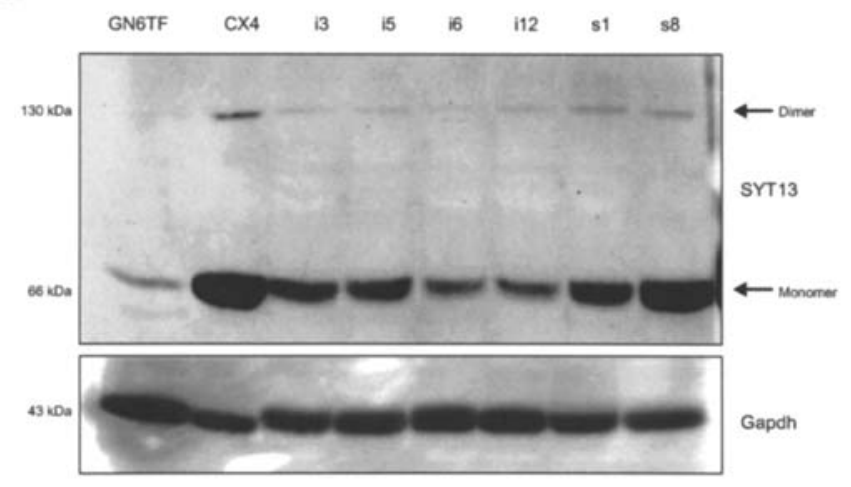

b

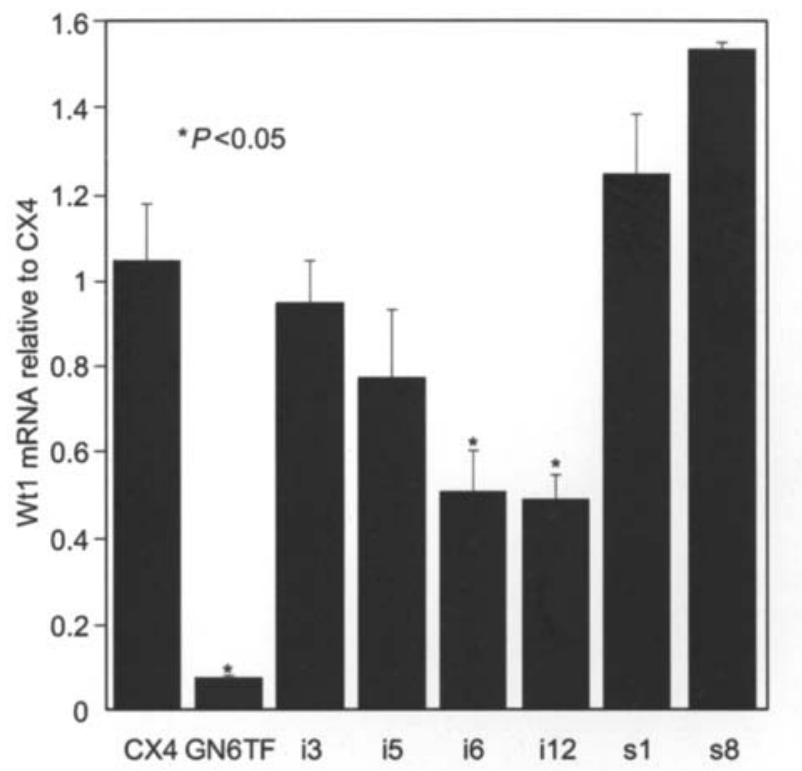

Figure 2. Molecular characterization of SYT13-i and SYT13-s cell lines (a) Western analysis of $S Y T 13$ protein expression in GN6TF, GN6TF$11^{\text {neo }} \mathrm{CX} 4$ microcell hybrid cells, SYT13-i and SYT13-s cell lines. The migration of the monomeric $(66 \mathrm{kDa})$ and dimeric $(130 \mathrm{kDa})$ forms of SYT13 are indicated. (b) Quantitative real-time RT-PCR analysis of Wt1 expression in SYT13-i and SYT13-s cell lines expressed relative to GN6TF$11^{\text {neo }} \mathrm{CX} 4$ cells. ${ }^{*} \mathrm{p}<0.05$ compared to GN6TF- $11^{\text {neo }} \mathrm{CX} 4$ cells using the oneway ANOVA.

observed in GN6TF-11 ${ }^{\text {neo }} \mathrm{CX} 4$ cells (data not shown), and the other (Isg20, interferon stimulated exonuclease 20) was modestly induced (2-fold). These results suggest that minimal interferon-associated gene expression changes accompany the transfection of SYT13-i or SYT13-s shRNA expression vectors in GN6TF-11 ${ }^{\text {neo }} \mathrm{CX} 4$ cells

siRNAs have been reported to affect translation of unintended transcripts containing partial complementarity to targets $(29,30)$. A BLAST search (http://www.ncbi.nlm. nih.gov/blast/) of the 21 nucleotide sequences corresponding to the SYT13-i target and SYT13-s scrambled control identified several potential off-target genes in the rat genome. The SYT13-i target matched 31 potential off-target genes with $14 / 21(67 \%)$ to $18 / 21(86 \%)$ sequence identity $(67 \%$, $\mathrm{n}=14 ; 71 \%, \mathrm{n}=8 ; 76 \%, \mathrm{n}=5 ; 81 \%, \mathrm{n}=1 ; 86 \%, \mathrm{n}=3)$. Likewise, the SYT13-s control matched 25 potential off-target genes with $67-86 \%$ sequence identity $(67 \%, \mathrm{n}=9 ; 71 \%, \mathrm{n}=5 ; 76 \%$, $\mathrm{n}=4 ; 81 \%, \mathrm{n}=6 ; 86 \%, \mathrm{n}=1)$. Overall, the SYT13-i and SYT13-s
shRNAs match 33 off-target genes with $>15 / 21(71 \%)$ sequence identity. However, microarray analysis failed to show significant alterations in mRNA levels of these potential off-target genes, suggesting minimal off-target effects from these shRNAs.

Molecular analysis of SYT13-i and SYT13-s cell lines. RNA samples corresponding to Zeocin-resistant SYT13-i and SYT13-s clones were analyzed by semi-quantitative and real-time RT-PCR to determine RNAi-mediated alterations in the expression of several genes, including human genes (PRDM11 and TP53I11) carried on human 11p11.2 (proximal to $S Y T 13$ ) and rat $W t 1$, which has been implicated in 11p11.2-mediated tumor suppression in our model system (14). SYT13, PRDM11 and TP53I11 are located within a $\sim 350 \mathrm{kbp}$ region of human $11 \mathrm{p} 11.2$. Semi-quantitative RTPCR analysis of PRDM11 and TP53I11 failed to detect any change in expression of these genes between GN6TF$11^{\text {neo }} \mathrm{CX} 4$ cells and the $S Y T 13-\mathrm{i}$ and $S Y T 13$-s cell lines analyzed (including SYT13-i3, SYT13-i5, SYT13-i6, SYT13i12, SYT13-s1 and SYT13-s8), suggesting that knockdown of SYT13 does not result in secondary alterations in the mRNA expression of these genes (data not shown).

Our previous studies identified a correlation between human 11p11.2-mediated rat liver tumor suppression and induction of rat Wtl (14). Based on this observation we hypothesized that the molecular mechanism governing rat liver tumor suppression by human $11 \mathrm{p} 11.2$ may directly or indirectly involve this previously identified tumor suppressor gene (14). Thus, if the microcell-mediated introduction of SYT13 into the cells is responsible for the induction of $W t 1$, we expect the expression of Wt1 to be diminished in SYT13attenuated cell lines. The parental GN6TF tumor cells express $W t 1$ at a significantly lower level than the suppressed GN6TF-1 $1{ }^{\text {neo }} \mathrm{CX} 4$ cells (Fig. 2b). Likewise, the level of $W t 1$ mRNA was significantly decreased in cell lines transfected with SYT13-i constructs, but was unchanged (or higher) in SYT13-s cell lines (Fig. 2b). The loss of Wt1 expression concurrent with attenuation of SYT13 was most pronounced in SYT13-i6 and SYT13-i12 (Fig. 2b). Furthermore, the expression of Wt1 significantly correlates with the level of SYT13 protein found in the SYT13-i and SYT13-s cell lines evaluated $(\mathrm{R}=0.79, \mathrm{p}=0.035)$. These data: i) support our previous hypothesis that expression of Wt1 is directly or indirectly regulated in response to $\mathrm{hSYT13}$ in this model system; and ii) suggest strongly that Wt1 is an important down-stream effector of SYT13-mediated tumor suppression.

Phenotypic and growth characteristic analyses of SYT13-i and SYT13-s cell lines in vitro. Analysis of morphology, contact inhibition, and anchorage-dependent growth in GN6TF- $11{ }^{\text {neo }} \mathrm{CX} 4$ cells revealed that human $11 \mathrm{p} 11.2$ normalizes the aggressive phenotype of the GN6TF cells in vitro $(13,14)$. After targeting human SYT13 in GN6TF$11^{\text {neo }} \mathrm{CX} 4$ cells with shRNAs, we re-examined these characteristics to assess the contribution of SYT13 to the suppression of the neoplastic cell phenotype of GN6TF cells in vitro. GN6TF cells are spindle-shaped and grow in multiple layers, whereas GN6TF- $11{ }^{\text {neo }} \mathrm{CX} 4$ cells exhibit a flattened and polygonal cell morphology and produce a 
a
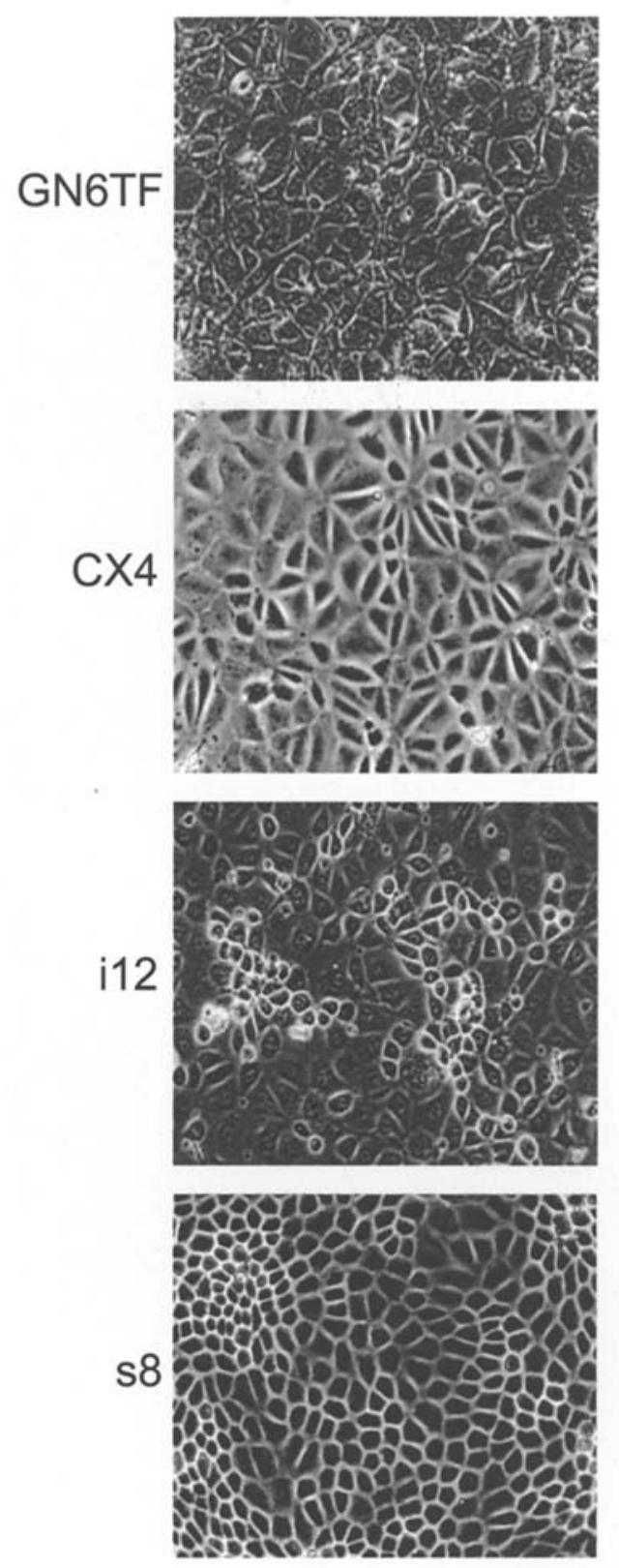

b

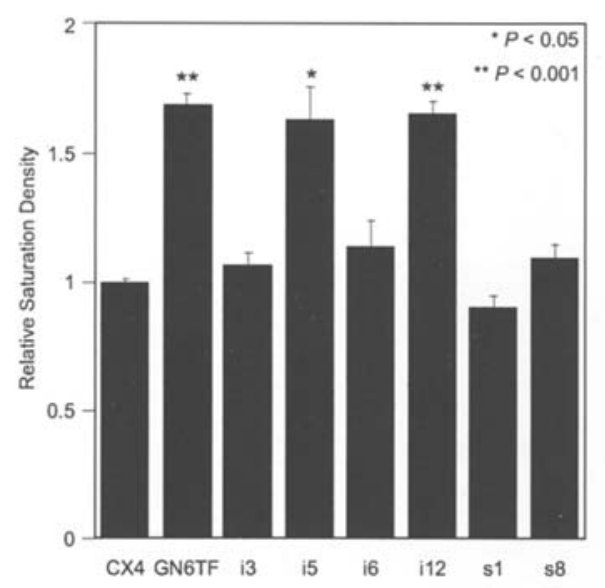

C

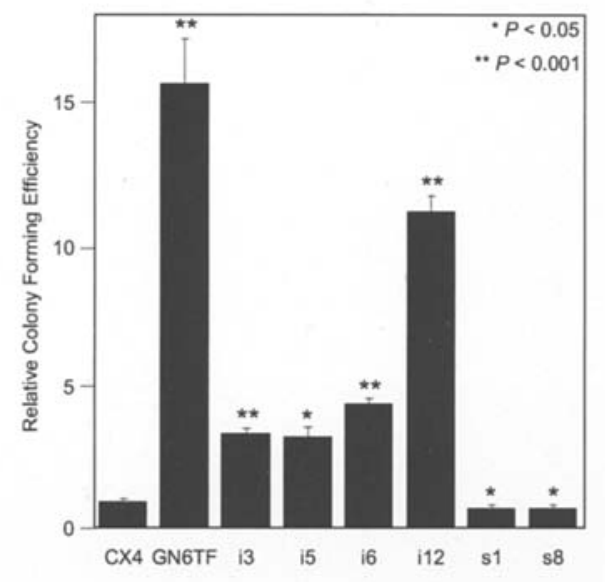

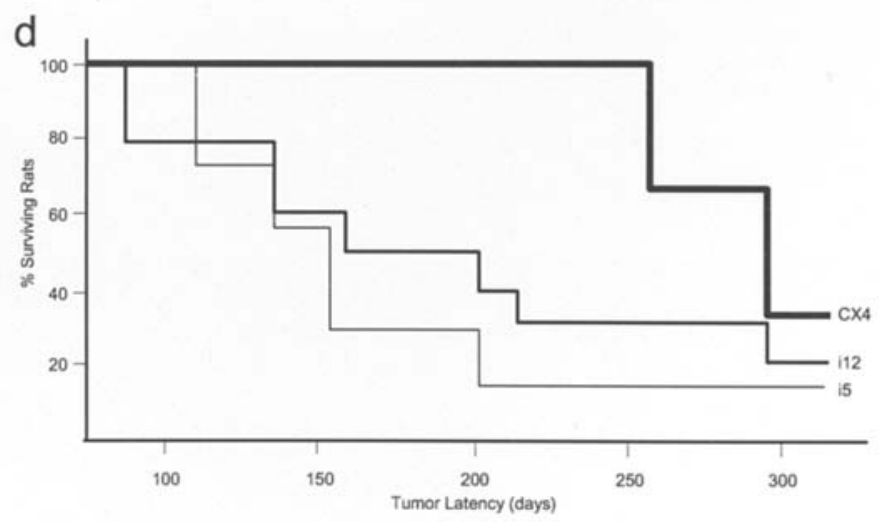

Figure 3. Phenotypic characterization of SYT13-i and SYT13-s cell lines. (a) Phase contrast microscopy of GN6TF tumor cells, GN6TF-11 ${ }^{\text {neo }}$ CX4 microcell hybrid cells, and representative derived SYT13-i and SYT13-s transfected cell lines. (b) Saturation densities of GN6TF tumor cells, GN6TF-11 ${ }^{\text {neo }}$ CX4 microcell hybrid cells, and representative derived SYT13-i and SYT13-s transfected cell lines (expressed relative to GN6TF-1 ${ }^{\text {neo }}$ CX4 cells). (c) Anchorageindependent growth of GN6TF cells, GN6TF-1 $1{ }^{\text {neo }} \mathrm{CX} 4$ microcell hybrid cells, and representative SYT13-i and SYT13-s transfected cell lines (expressed relative to CX4 cells). (d) Kaplan-Meier analysis of survival for animals transplanted with GN6TF-1 $1{ }^{\text {neo }} \mathrm{CX} 4$ cells, SYT13-i12 and SYT13-i5 cell lines. ${ }^{*} \mathrm{p}<0.05$ and ${ }^{* *} \mathrm{p}<0.001$ compared to GN6TF-1 $1{ }^{\text {neo }} \mathrm{CX} 4$ cells using Student's t-test (unpaired data with unequal variance); $\mathrm{p}$-values for (d) are compared to GN6TF- $11^{\text {neo }} \mathrm{CX} 4$ cells using the log-rank test.

contact-inhibited growth pattern (Fig. 3a). SYT13-i cell lines retain some polygonal features similar to $\mathrm{GN} 6 \mathrm{TF}-11^{\text {neo }} \mathrm{CX} 4$ cells, but exhibit a multilayer growth pattern after reaching confluence in culture (Fig. 3a). The morphologies of controltransfected cell lines (SYT13-s1 and SYT13-s8) were similar to that of the parental suppressed cell line GN6TF-11 ${ }^{\text {neo }} \mathrm{CX} 4$ (Fig. 3a). Fig. 3b shows the saturation densities of the SYT13-i and SYT13-s cell lines analyzed. SYT13-i5 and SYT13-i12 grow to an elevated saturation density that is indistinguishable from GN6TF, reflecting multilayer growth. In contrast, the saturation densities of SYT13-s1 and SYT13-s8 were indistinguishable from that of the GN6TF- $11^{\text {neo }} \mathrm{CX} 4$ cells. Fig. 3c shows the anchorage-independent growth potential of the SYT13-i and SYT13-s cell lines analyzed. All of the SYT13-i cell lines efficiently produced colonies in soft agarose, indicative of the restoration of anchorage- 
Table I. Tumorigenic potential of GN6TF-11 ${ }^{\text {neo }} \mathrm{CX} 4$ and derived SYT13-i and SYT13-s cell lines following transplantation into neonatal Fischer 344 rats.

\begin{tabular}{|c|c|c|c|c|c|}
\hline Cell Line & $\begin{array}{c}\text { Tumorigenicity }{ }^{\mathrm{a}} \\
(\%)\end{array}$ & $\begin{array}{l}\text { Average latency } \\
\text { (days) }\end{array}$ & $\begin{array}{l}\text { Latency range } \\
\text { (days) }\end{array}$ & $\begin{array}{l}\text { Latency significance } \\
\qquad(\mathrm{P})^{\mathrm{b}}\end{array}$ & $\begin{array}{c}\text { Survival significance } \\
(\mathrm{P})^{\mathrm{c}}\end{array}$ \\
\hline GN6TF-1 $11^{\text {neo }} C X 4$ & $4 / 6(67)$ & $274 \pm 11$ & $256-293$ & - & - \\
\hline SYT13-i3 & $1 / 5(20)$ & 201 & $\mathrm{NA}^{\mathrm{d}}$ & NA & $0.10(\mathrm{NS})^{\mathrm{e}}$ \\
\hline SYT13-i5 & $6 / 7(86)$ & $144 \pm 14$ & $110-201$ & $<0.0001$ & 0.04 \\
\hline SYT13-i6 & $1 / 8(12)$ & 201 & NA & NA & 0.07 (NS) \\
\hline SYT13-i12 & $8 / 10(80)$ & $164 \pm 25$ & $87-293$ & 0.002 & 0.15 (NS) \\
\hline SYT13-s1 & $1 / 4(25)$ & 256 & NA & NA & 0.28 (NS) \\
\hline SYT13-s8 & $3 / 4(75)$ & $216 \pm 41$ & $153-293$ & $0.28(\mathrm{NS})$ & 0.48 (NS) \\
\hline
\end{tabular}

${ }^{a}$ Tumorigenic potential is expressed as the percentage of animals forming tumors per total animals receiving cell transplants. ${ }^{b} \mathrm{Compared}$ to GN6TF- $11^{\text {neo }} \mathrm{CX} 4$ cells using the Student's t-test. ${ }^{\mathrm{c} C o m p a r e d}$ to GN6TF-1 $1{ }^{\text {neo }} \mathrm{CX} 4$ cells using the log-rank test. ${ }^{\mathrm{d}}$ Not applicable; ${ }^{\mathrm{e}}$ not significant.

independent growth potential (Fig. 3c). SYT13-i12 formed the most soft agarose colonies, comparable to the number observed with GN6TF cells. In contrast, SYT13-s 1 and SYT13-s8 cells formed few colonies in soft agarose, similar to GN6TF-1 $11^{\text {neo }} \mathrm{CX} 4$ cells (Fig. 3c). These results combine to suggest that several cellular features of suppressed GN6TF$11^{\text {neo }} \mathrm{CX} 4$ microcell hybrid cells (including growth pattern, contact inhibition and anchorage-dependent growth) correlate with expression of $S Y T 13$, and may be directly or indirectly regulated by $S Y T 13$.

Tumorigenic potential of SYT13-i and SYT13-s cell lines in vivo. Several SYT13-i and SYT13-s cell lines were selected for analysis of tumorigenicity in vivo. SYT13-i5 and SYT13-i12 cells displayed the greatest tumorigenic potential with 86 and $80 \%$ of syngeneic animals (respectively) developing tumors with short latency (Table I). In contrast, the suppressed parental GN6TF-1 $1{ }^{\text {neo }} \mathrm{CX} 4$ cells developed fewer tumors with a much longer average latency, consistent with previous studies (8). The average latency for tumor formation by SYT13-s1 and SYT13-s8 cells in the 50\% (4/8) of animals which developed tumors was $226 \pm 31$ days, similar to the GN6TF- $11^{\text {neo }} \mathrm{CX} 4$ cells (Table I). The increased aggressiveness of SYT13-i cell lines was evident from a KaplanMeier analysis of survival after transplantation of SYT13-i cell lines (Fig. 3d). This analysis revealed a statistically significant survival disadvantage for animals transplanted with SYT13-i5 cells ( $\mathrm{p}=0.04)$ compared to GN6TF-1 $1{ }^{\text {neo }} \mathrm{CX} 4$ cells (Fig. 3d). Likewise, a trend suggestive of survival disadvantage was observed among animals transplanted with SYT13-i12 cells (compared to GN6TF-11 ${ }^{\text {neo }} \mathrm{CX} 4$ cells), but the trend did not reach significance $(\mathrm{p}=0.15)$.

\section{Discussion}

Synaptotagmin XIII is an atypical member of the synaptotagmin family of membrane-associated synaptic vesicle transport proteins which largely serve as $\mathrm{Ca}^{2+}$ sensors in vesicular trafficking and exocytosis $(22,31)$. The function of SYT13 is unknown, but appears to lack some conserved functions of other family members $(22,23)$, including calciumdependent phospholipid binding in vesicle transport and calcium-independent binding to target SNARE heterodimers during calcium-triggered membrane fusion (32). We have identified a potential role for SYT13 in liver tumor suppression. The human chromosome 11 liver tumor suppressor locus was identified through deletion mapping of microcell hybrid cell lines derived from GN6TF rat liver tumor cells (9). The minimal liver tumor suppressor region of human 11 p11.2 contains a number of potential candidate tumor suppressor genes (10-12). However, transcription mapping of suppressed and non-suppressed microcell hybrid cell lines reduced the number of viable candidate genes and produced evidence suggesting that SYT13 is the best candidate for the human $11 \mathrm{p} 11.2$ liver tumor suppressor (11). hSYT13 is expressed in suppressed microcell hybrid cell lines, but not in non-suppressed microcell hybrid cell lines (11). Additionally, hSYT13 was not expressed in one microcell hybrid cell line that was determined to have an interstitial deletion in the human 11 p 11.2 liver tumor suppressor region $(11,13)$. Thus, our studies indicate that expression of hSYT13 accompanies 11p11.2-mediated liver tumor suppression, strongly suggesting that SYT13 contributes directly or indirectly to tumor suppression in this model system. GN6TF cells are highly tumorigenic in vivo (100\% of host animals develop tumors with short latency) and exhibit aggressive phenotypic characteristics in vitro, including loss of contact inhibition, growth to elevated saturation density and anchorage-independent growth $(15,33)$. Suppression of the neoplastic phenotype of GN6TF cells by introduction of human chromosome 11 results in reduced tumorigenic potential (fewer tumors formed with longer latency), and normalization of the cell phenotype in vitro (restoration of contact inhibition, reduction of 


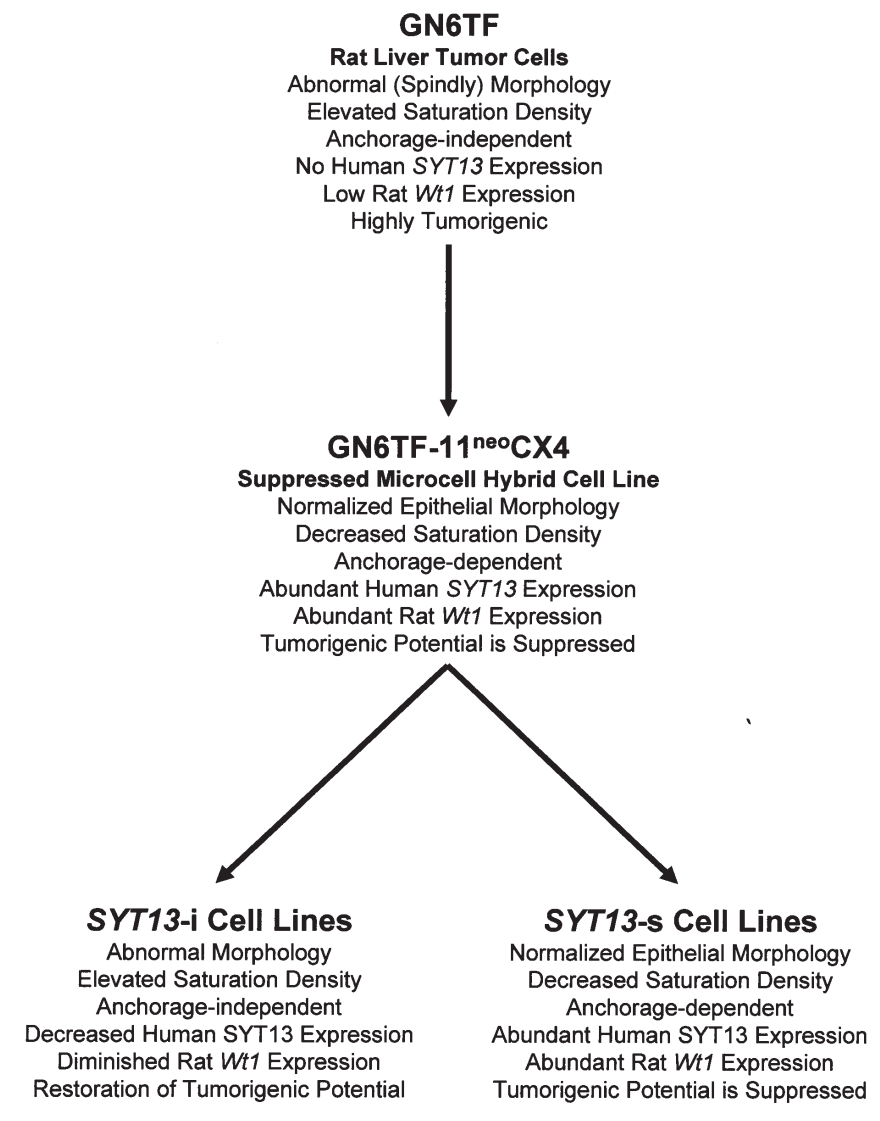

Figure 4. Evidence for SYT13-mediated tumor suppression in GN6TF rat liver tumor cells. The major phenotypic properties of GN6TF tumor cells, GN6TF-1 $11^{\text {neo }} \mathrm{CX} 4$ microcell hybrid cells, and derived SYT13-i and SYT13-s cell lines are summarized. In addition, the relative gene expression levels of SYT13 and Wt1 are indicated for each cell type.

saturation density, and loss of anchorage-independent growth potential) (8). As summarized in Fig. 4, when human $11 \mathrm{p} 11.2$ is introduced into GN6TF cells (resulting in suppressed microcell hybrid cell lines), hSYT13 is expressed coordinate with suppression of tumorigenicity and normalization of the cellular phenotype. In previous studies, re-expression of tumorigenicity in suppressed microcell hybrid cell lines followed loss of the suppressive human 11 p11.2 chromosome (8), and the related loss of hSYT13 expression (11). In the current study, we show that RNAimediated silencing of SYT13 results in re-expression of the neoplastic phenotype of GN6TF tumor cells (Fig. 4). This result suggests strongly that $S Y T 13$ directly or indirectly regulates the growth properties of these cells in vitro and that loss of SYT13 significantly contributes to tumorigenic potential in vivo. GN6TF tumor cells express the monomeric species of rSyt13 at easily detectable levels (Fig. 2a), but this protein fails to form dimers. Expression of exogenous $\mathrm{h} S Y T 13$ (from human $11 \mathrm{p} 11.2$ ) results in the production of both monomeric protein and dimeric complexes (Fig. 2a). In the presence of a dimeric form of SYT13, a molecular defect in GN6TF tumor cells is complemented (and corrected) by a gene from human $11 \mathrm{p} 11.2$, resulting in suppression of tumorigenicity. These observations suggest that: i) the endogenous rSyt13 may be defective or non-functional, possibly as a consequence of a missense mutation in the $\mathrm{rSyt} 13$ gene; ii) the dimeric form of the rSyt13 protein is required for expression of its tumor suppressive functions; and iii) the endogenous $\mathrm{rSyt} 13$ in GN6TF cells is unable to dimerize, resulting in loss of tumor suppressor function.

The molecular mechanism for human 11p11.2-mediated liver tumor suppression has not yet been fully elucidated. However, our previous studies identified a precise correlation between Wt1 expression and suppression of tumorigenicity among microcell hybrid cell lines, suggesting that the molecular mechanism governing human 11p11.2-mediated liver tumor suppression may directly or indirectly involve the induction of rat Wt1 (14). The current study supports this hypothesis. In response to RNAi-mediated attenuation of SYT13, Wt1 expression levels decline coordinate with reexpression of the neoplastic phenotype. This observation suggests a mechanistic linkage between SYT13 and Wt1. The function of SYT13 is unknown. However, Fukuda and Mikoshiba have suggested that this membrane-associated protein is involved in a constitutive pathway that docks transport vesicles to plasma membranes (22). Thus, SYT13 may function in cellular signaling, relaying extracellular signals to the nucleus and eventually contributing to the regulation of transcription. The current study suggests that the rSyt13 expressed in GN6TF cells may be defective (nonfunctional) as a consequence of some as yet unidentified mutation that results in a failure (or inability) of the protein to dimerize. It is intriguing to speculate that the loss of rSyt13 function interrupts normal cell signaling, resulting in loss of expression of other genes, such as Wt1. It follows that expression of a functional hSYT13 in these cells would restore normal signaling, resulting in re-expression of $W t 1$. However, additional studies will be required to establish a direct linkage between SYT13 (either the monomeric or dimeric form) and regulation of Wt1 transcription.

\section{Acknowledgements}

This work was supported by grants from the National Cancer Institute (NIH grant CA078434), and the University Research Council of the University of North Carolina at Chapel Hill. J.E.J. was supported, in part, by NIH training grant T32 ES07017. The authors would like to acknowledge Dr Hyung-Suk Kim, Department of Pathology and Laboratory Medicine, UNC School of Medicine, for his cogent insight and advice regarding real-time RT-PCR design, application and interpretation and Dr Wendell D. Jones, Expression Analysis, Durham, NC, for his manipulation and analysis of microarray-generated data.

\section{References}

1. Grisham JW: Molecular genetic alterations in primary hepatocellular neoplasms. In: The Molecular Basis of Human Cancer. Coleman WB and Tsongalis GJ (eds). Humana Press, Totowa, NJ, pp269-346, 2002.

2. Thorgeirsson SS and Grisham JW: Molecular pathogenesis of human hepatocellular carcinoma. Nat Genet 31: 339-346, 2002.

3. Boige V, Laurent-Puig P, Fouchet P, et al: Concerted nonsyntenic allelic losses in hyperploid hepatocellular carcinoma as determined by a high-resolution allelotype. Cancer Res 57: 1986-1990, 1997.

4. Nagai H, Pineau P, Tiollais P, et al: Comprehensive allelotyping of human hepatocellular carcinoma. Oncogene 14: 2927-2933, 1997. 
5. Piao Z, Park C, Park JH and Kim H: Allelotype analysis of hepatocellular carcinoma. Int J Cancer 75: 29-33, 1998.

6. Fujimori M, Tokino T, Hino $\mathrm{O}$, et al: Allelotype study of primary hepatocellular carcinoma. Cancer Res 51: 89-93, 1991.

7. Grisham JW: Interspecies comparison of liver carcinogenesis: implications for cancer risk assessment. Carcinogenesis 18: 59-81, 1997.

8. Coleman WB, McCullough KD, Esch GL, et al: Suppression of the tumorigenic phenotype of a rat liver epithelial tumor cell line by the p11.2-p12 region of human chromosome 11 . Mol Carcinog 13: 220-232, 1995.

9. Coleman WB, Esch GL, Borchert KM, et al: Localization of a putative liver tumor suppressor locus to a $950-\mathrm{kb}$ region of human 11p11.2-p12 using rat liver tumor microcell hybrid cell lines. Mol Carcinog 19: 267-272, 1997.

10. Ricketts SL, Garcia NF, Betz BL and Coleman WB: Identification of candidate liver tumor suppressor genes from human 11p11.2-p12. Genes Chromosomes Cancer 33: 47-59, 2002.

11. Jahn JE, Ricketts SL and Coleman WB: Identification of candidate liver tumor suppressor genes from human $11 \mathrm{p} 11.2$ by transcription mapping of microcell hybrid cell lines. Int $\mathrm{J}$ Oncol 22: 1303-1310, 2003.

12. Ricketts SL, Carter JC and Coleman WB: Identification of three $11 \mathrm{p} 11.2$ candidate liver tumor suppressors through analysis of known human genes. Mol Carcinog 36: 90-99, 2003.

13. Mahon MC, Driscoll MP, Glover WJ, et al: Suppression of tumorigenicity of rat liver epithelial tumor cell lines by a putative human 11p11.2-p12 liver tumor suppressor locus. Int J Oncol 14: 337-346, 1999.

14. Coleman WB, Ricketts SL, Borchert KM, et al: Induction of rat WT1 gene expression correlates with human chromosome $11 \mathrm{p} 11.2$-p12-mediated suppression of tumorigenicity in rat liver epithelial tumor cell lines. Int J Oncol 14: 957-963, 1999.

15. Tsao MS and Grisham JW: Phenotypic modulation during tumorigenesis by clones of transformed rat liver epithelial cells. Cancer Res 47: 1282-1286, 1987.

16. Tsao MS, Smith JD, Nelson KG and Grisham JW: A diploid epithelial cell line from normal adult rat liver with phenotypic properties of 'oval' cells. Exp Cell Res 154: 38-52, 1984.

17. Saxon PJ, Srivatsan ES, Leipzig GV, Sameshima JH and Stanbridge EJ: Selective transfer of individual human chromosomes to recipient cells. Mol Cell Biol 5: 140-146, 1985.

18. Banerjee A, Xu HJ, Hu SX, et al: Changes in growth and tumorigenicity following reconstitution of retinoblastoma gene function in various human cancer cell types by microcell transfer of chromosome 13. Cancer Res 52: 6297-6304, 1992.
19. Lee LW, Raymond VW, Tsao MS, Lee DC, Earp HS and Grisham JW: Clonal cosegregation of tumorigenicity with overexpression of c-myc and transforming growth factor alpha genes in chemically transformed rat liver epithelial cells. Cancer Res 51: 5238-5244, 1991.

20. Rozen S and Skaletsky H: Primer3 on the WWW for general users and for biologist programmers. Methods Mol Biol 132: 365-386, 2000.

21. Rivenbark AG, Jones WD, Risher JD and Coleman WB: DNA methylation-dependent epigenetic regulation of gene expression in MCF-7 breast cancer cells. Epigenetics 1: 32-44, 2006.

22. Fukuda M and Mikoshiba K: Characterization of KIAA1427 protein as an atypical synaptotagmin (Syt XIII). Biochem J 354: 249-257, 2001.

23. Von Poser C and Sudhof TC: Synaptotagmin 13: structure and expression of a novel synaptotagmin. Eur J Cell Biol 80: 41-47, 2001.

24. Fukuda M, Kanno E, Ogata Y and Mikoshiba K: Mechanism of the SDS-resistant synaptotagmin clustering mediated by the cysteine cluster at the interface between the transmembrane and spacer domains. J Biol Chem 276: 40319-40325, 2001.

25. Mise-Omata S, Obata Y, Iwase S, Mise N and Doi TS: Transient strong reduction of PTEN expression by specific RNAi induces loss of adhesion of the cells. Biochem Biophys Res Commun 328: 1034-1042, 2005.

26. Bridge AJ, Pebernard S, Ducraux A, Nicoulaz AL and Iggo R: Induction of an interferon response by RNAi vectors in mammalian cells. Nat Genet 34: 263-264, 2003.

27. Sledz CA, Holko M, De Veer MJ, Silverman RH and Williams BR: Activation of the interferon system by shortinterfering RNAs. Nat Cell Biol 5: 834-839, 2003.

28. Jackson AL and Linsley PS: Noise amidst the silence: off-target effects of siRNAs? Trends Genet 20: 521-524, 2004.

29. Scacheri PC, Rozenblatt-Rosen O, Caplen NJ, et al: Short interfering RNAs can induce unexpected and divergent changes in the levels of untargeted proteins in mammalian cells. Proc Natl Acad Sci USA 101: 1892-1897, 2004.

30. Saxena S, Jonsson ZO and Dutta A: Small RNAs with imperfect match to endogenous mRNA repress translation. Implications for off-target activity of small inhibitory RNA in mammalian cells. J Biol Chem 278: 44312-44319, 2003.

31. Fernandez-Chacon R, Konigstorfer A, Gerber SH, et al: Synaptotagmin I functions as a calcium regulator of release probability. Nature 410: 41-49, 2001.

32. Rickman C, Craxton M, Osborne $S$ and Davletov B: Comparative analysis of tandem $\mathrm{C} 2$ domains from the mammalian synaptotagmin family. Biochem J 378: 681-686, 2004

33. Tsao MS and Grisham JW: Hepatocarcinomas, cholangiocarcinomas, and hepatoblastomas produced by chemically transformed cultured rat liver epithelial cells. A light- and electron-microscopic analysis. Am J Pathol 127: 168-181, 1987. 